\title{
The Effect of Spiritual Care on Sleep Quality in Patients with Multiple Sclerosis referred to the MS Society of Hamadan city in 2018
}

\author{
Nasim Motavakel ${ }^{1}$, Zahra Maghsoudi², Younes Mohammadi ${ }^{3}$, Khodayar Oshvandi ${ }^{*}$
}

1. MSc. Student of Medical Surgical Nursing, Student Research Committee, School of Nursing and Midwifery, Hamadan University of Medical Sciences, Hamadan, Iran Corresponding Author, Contact Number: 09185851956

2. PhD Student in Nursing, Student Research Committee, Hamadan University of Medical Sciences, Hamadan, Iran

3. Assisstant Professor of Epidemiology, Modeling of Noncommunicable Diseases Research Center, Hamadan University of Medical Sciences, Hamadan, Iran

4. Professor, Medical Surgical Nursing, Mother and Child Care Research Center, Hamadan University of Medical Sciences, Hamadan, Iran

\section{Article Info}

Received: 2019/09/07;

Accepted: 2019/09/29;

Published Online: 2020/04/07

$\underline{10.30699 / a j n m c .28 .1 .36}$

Original Article

Use your device to scan and read the article online

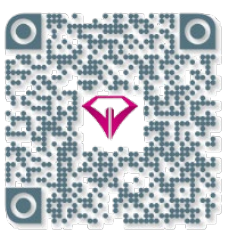

\section{ABSTRACT}

Introduction: Sleep has a soothing effect on the health and well-being of patients with multiple sclerosis (MS). It seems that nurses have an important task in improving their condition. Therefore, this study was designed to determine the effect of spiritual care on sleep quality in patients with MS.

Methods: In this semi-experimental study,70 patients with multiple sclerosis who referred to the MS Society of Hamadan were selected as available and randomly divided into two groups of control and experimental (each group included 35). Data were collected through a demographic questionnaire and Pittsburgh Sleep Quality. The intervention was held in the form of group meetings during the 4 weekly sessions of 45 to 60 minutes. Data were analyzed by SPSS 16 and descriptive and inferential statistics (independent t-test and paired t-test).

Results: There was no significant difference between the two groups in terms of demographic variables $(P>0.05)$. There was no significant difference between the two groups before intervention in the sleep quality variable $(P=0.678)$. There was a statistically significant difference between the two groups in the after-interventionphase $(P<0.001)$. Findings of the study showed the effectiveness of spiritual care on improving the quality of sleep in patients with multiple sclerosis.

Conclusion: Regarding the effect of spiritual care on improving the quality of sleep, it is suggested that nurses and health care providers pay attention to the spiritual needs of patients and use spiritual care as a safe and cost effective way to improve patients' sleep.

Keywords: Spiritual care, Sleep quality, Multiple Sclerosis

\section{How to Cite This Article:}

Motavakel N, Maghsoudi Z, Mohammadi Y, Oshvandi K. The Effect of Spiritual Care on Sleep Quality in Patients with Multiple Sclerosis referred to the MS Society of Hamadan city in 2018. Avicenna J Nurs Midwifery care. 2020; 28 (1) :36-45 
تأثير مراقبت معنوى بر كيفيت خواب بيماران مبتلا به مولتييل اسكلروزيس مراجعهكننده به انجمن ام اس لإ

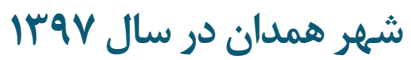

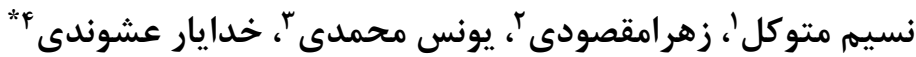

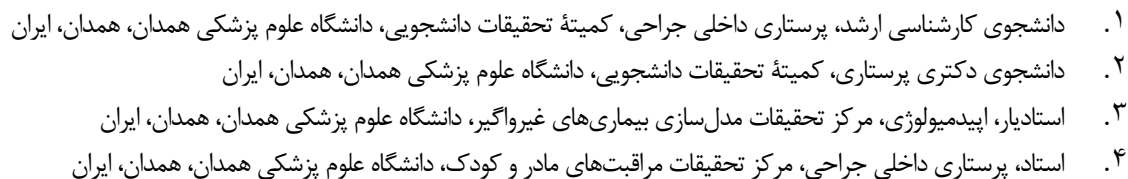

\begin{tabular}{|c|c|}
\hline "جكيده & اطلاعات مقاله \\
\hline 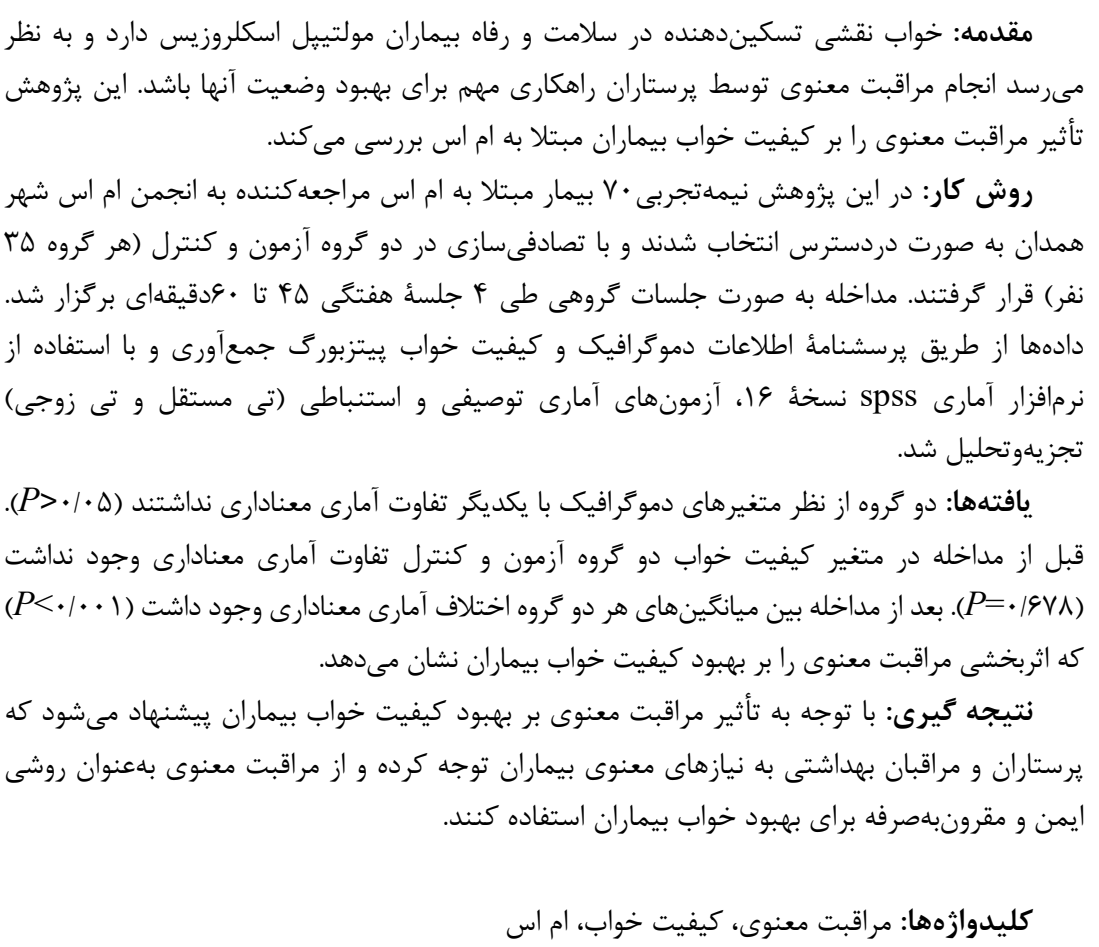 & 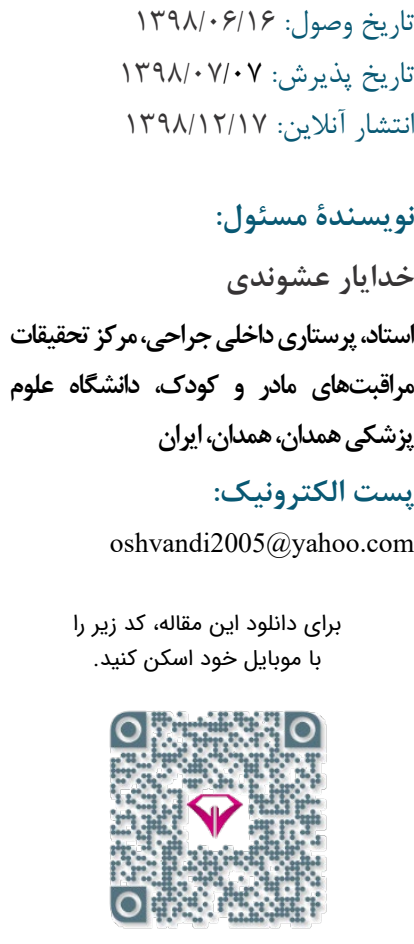 \\
\hline
\end{tabular}

مقدمه

خواب، افسردگى و اضطراب را دارند [ع ،ه ، ، [1]. كاهش كيفيت خواب از عوارض شايع در بيماران ام اس است؛ به رابه

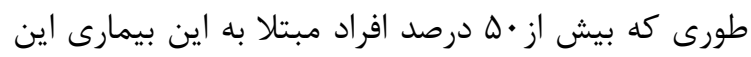

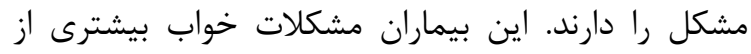
جمعيت عمومى و همينطور از ساير بيمارىهاى مزمن دارند

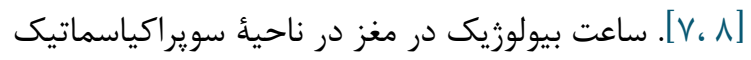

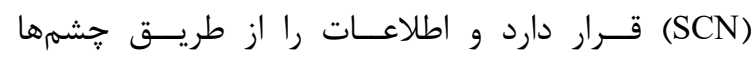

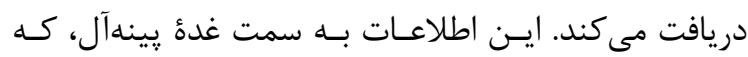
در ناحيـهُ ايى تالاموس واقع شده، حركت مسى كنـد. زمانى
مولتييل اسكلروزيس ] شايعترين بيمارى مزمن سيستم عصبى مركزى است [1] كه شيوع آن بهسرعت در حال ييشرفت است؛ بيه طورى كن كه

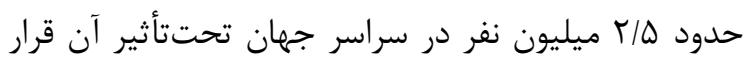

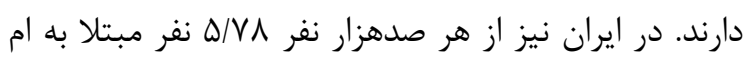

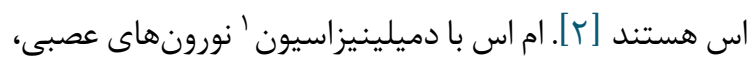

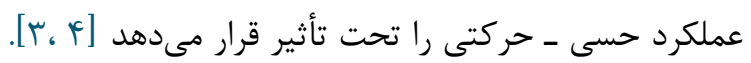

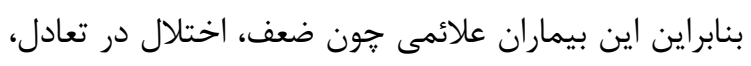
اختلال بينايى، كاهش توانايى حل مشكل، كاهش كيفيت

\footnotetext{
${ }^{1}$ Demyelination
} 
مناسب سيستمهاى فيزيولوزيكى منجر مىشود كه بيماران

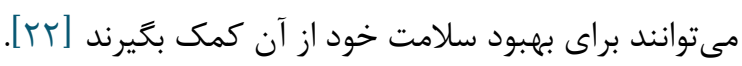

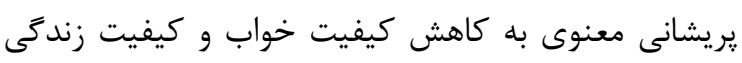

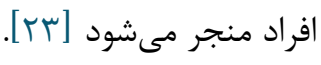
بنابر آنجه بيان شد بيماران مبتلا به ام ام اس كيفيت خواب

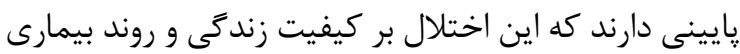

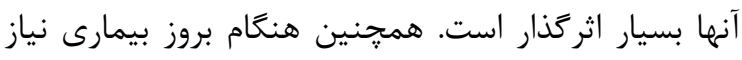

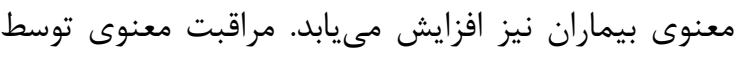

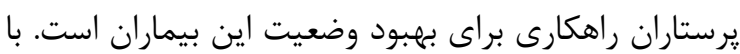

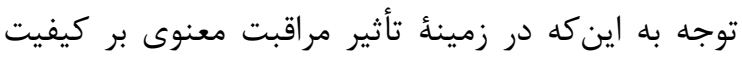

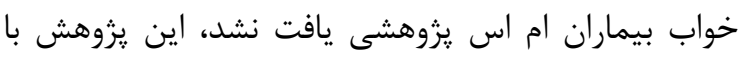

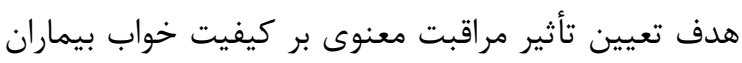

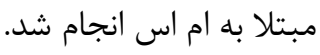

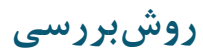

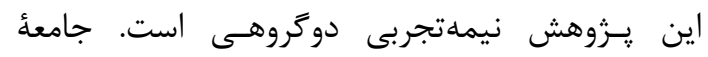

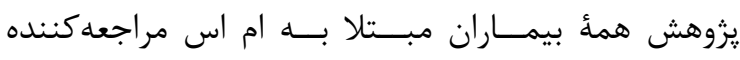

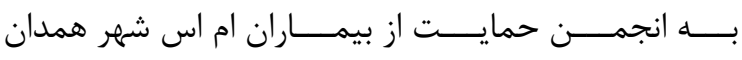

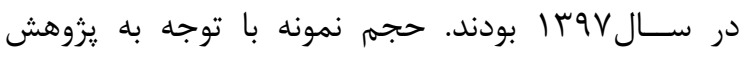
Taheri

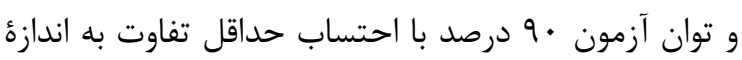

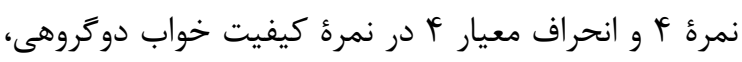

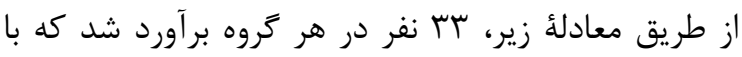

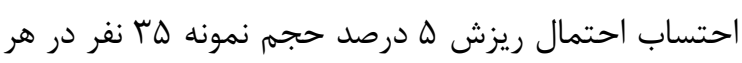
كروه تعيين شد.

$$
n_{1}=2 \frac{\left(Z_{\alpha}+Z_{\beta}\right)^{2} \sigma^{2}}{\Delta^{2}}
$$

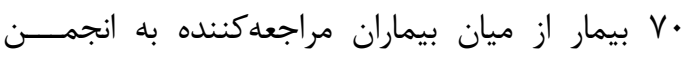

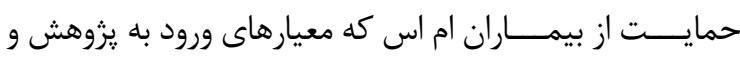

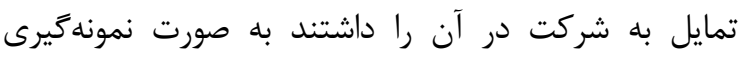

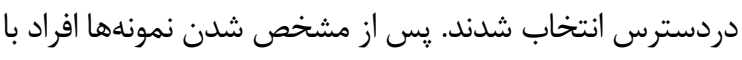

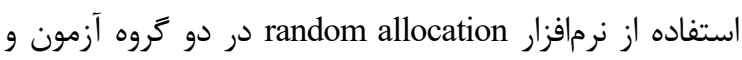
كنترل جاى كرفتند؛ به طورى كه هركدام از كروهها بهتفكيك

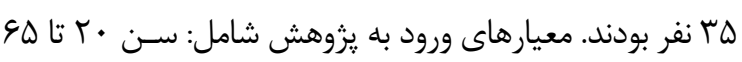

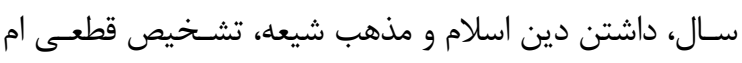
اس طبق نظر يزشك متخصص نورولوزيست، سطح ناتوانى بين

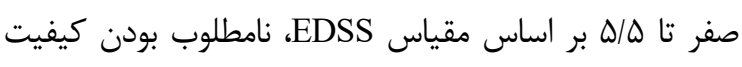

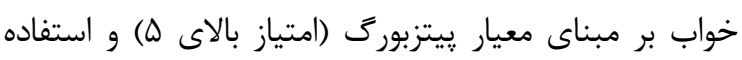

كه آسيبى به اين هستهها وارد شود ريتم شبانهروزى خواب

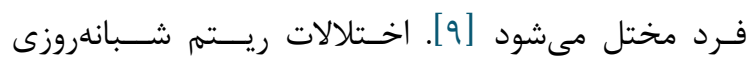

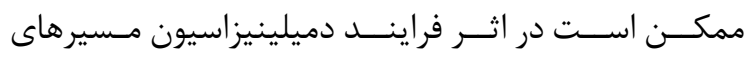

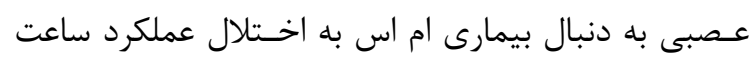

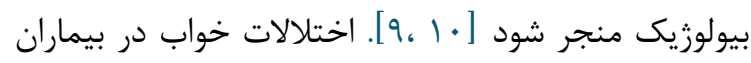

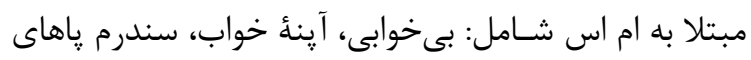

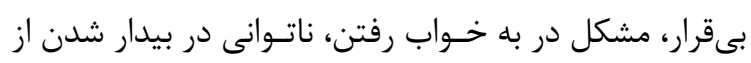

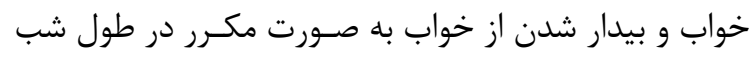

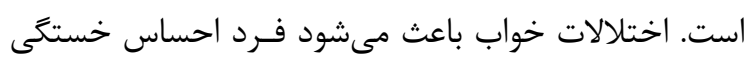

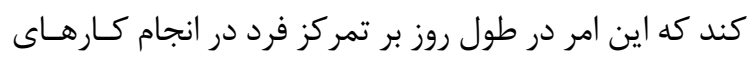

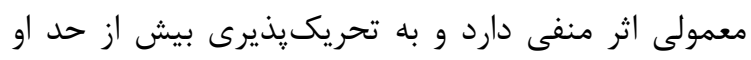

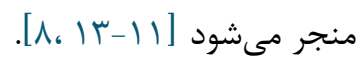
از رويكردهاى غيردارويى كه شايد بتواند روى كيفيت

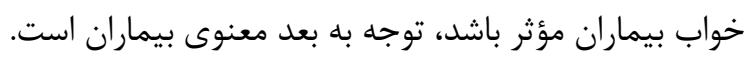

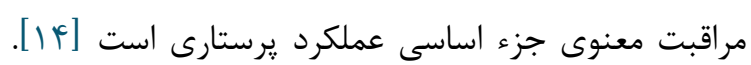

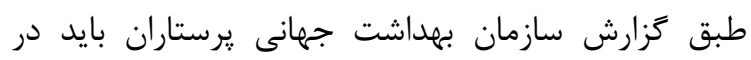

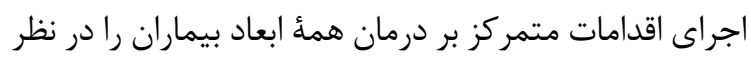

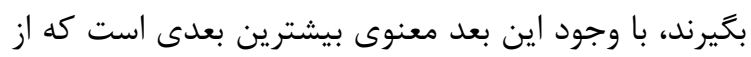

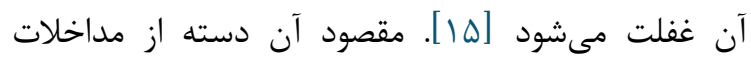

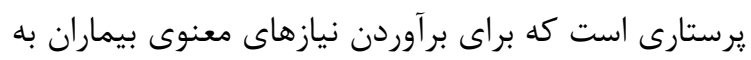

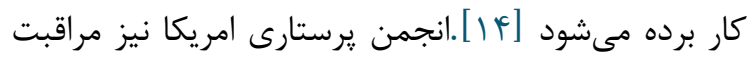

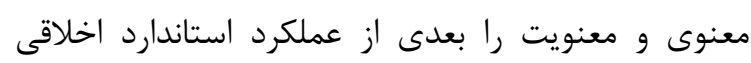

$$
\text { يرستارى تعريف مى كند [19، IV] }
$$
يزوهش ها نشان مى دهد كه دخالت شاخص مهاى مختلف

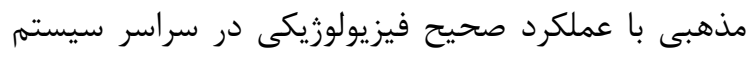

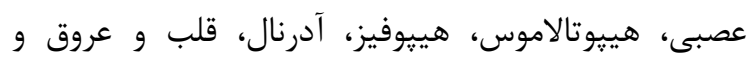

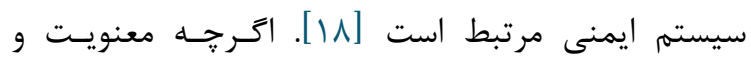

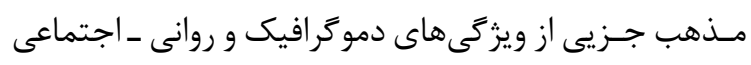

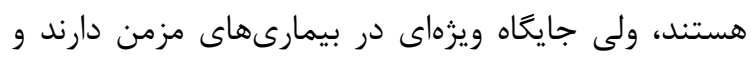

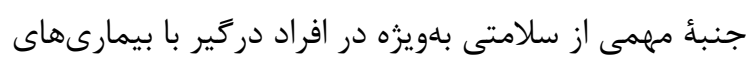
مزمن محسوب مىشوند [19]

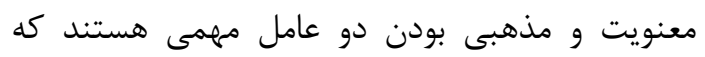

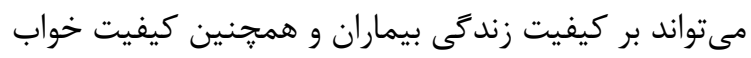

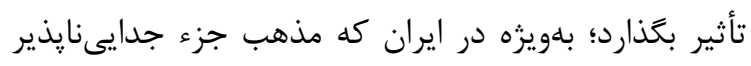

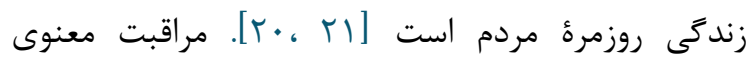

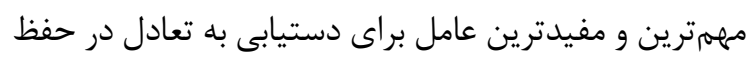

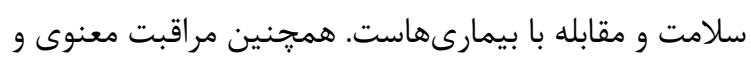
انجام تعاليم مذهبى با ايجاد احساسات مثبت به عملكرد 
طراحى و نهايى شد [ro]]. اين يرسشنامه در ايران نيز ابزارى

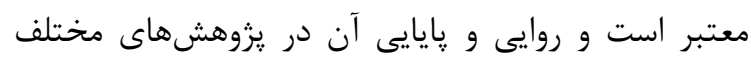

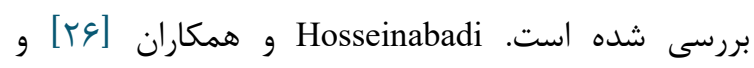
Soleimany

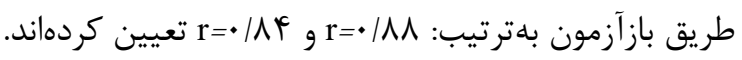

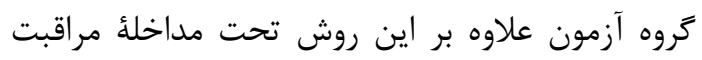

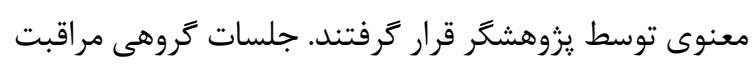

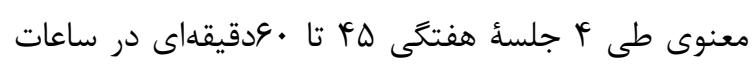

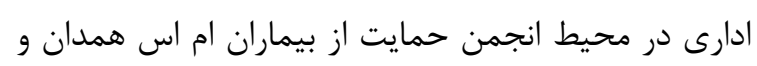

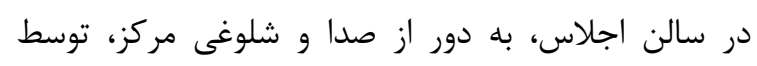

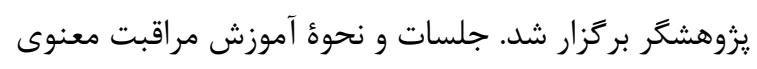

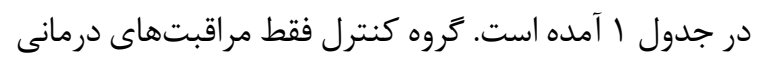

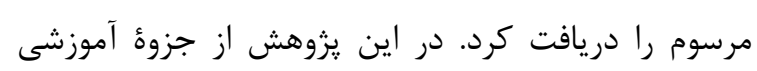

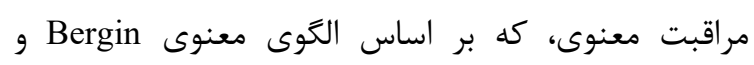
Richards

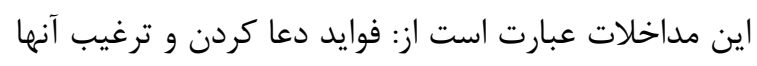

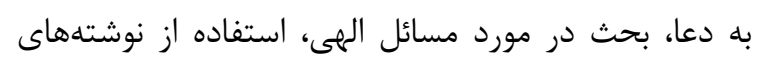

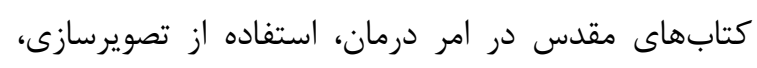

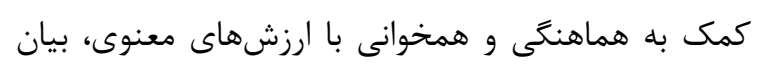

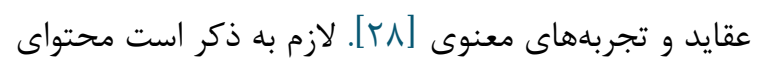

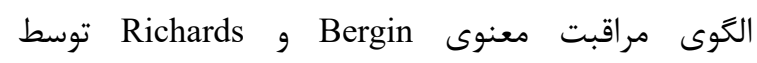
كارشناسان مذهبى و اساتيد دانشعاه علوم بهزيستى و و

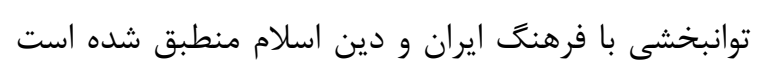

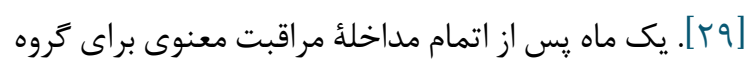

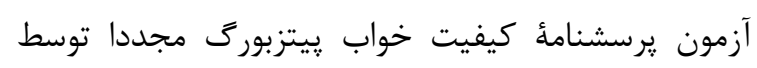

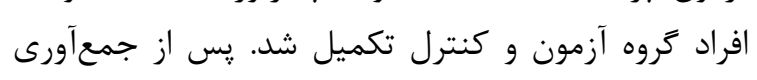

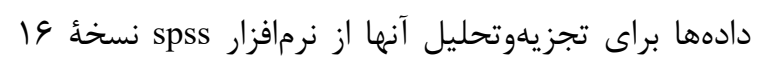
(SPSS Inc., Chicago, Ill., USA) توصيفى (ميانكين و انحراف معيار) و استنباطى (مانند تى آنى

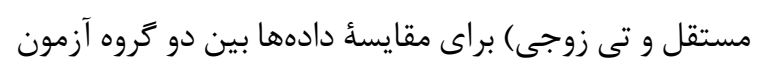

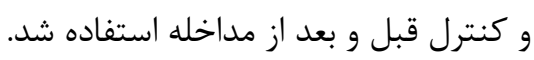

نكردن از داروهاى خوابآور بود. معيارهاى خروج از يزوهش

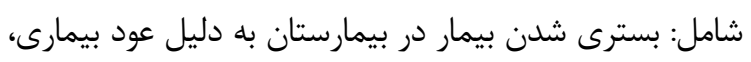

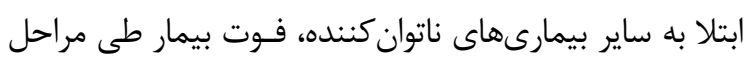

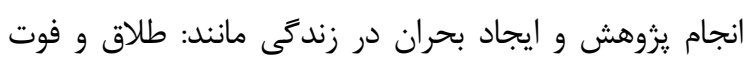
والدين بود. قبل از مداخله فرم رضايت آكاهانه و يرسشنامه براى

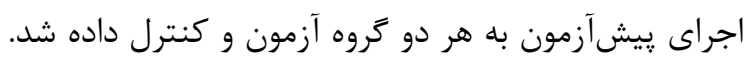

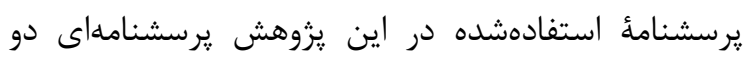

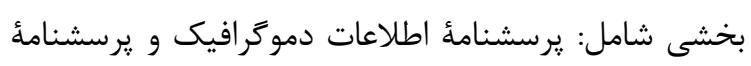

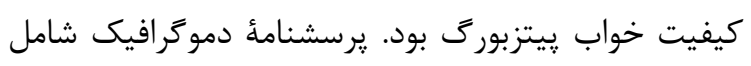

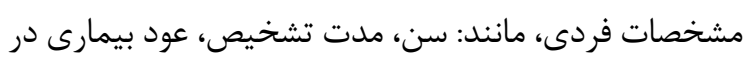

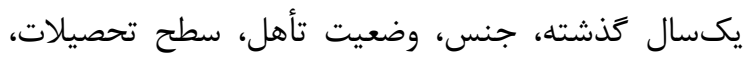

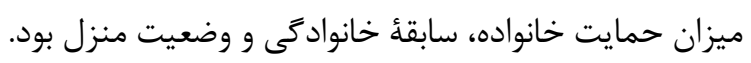

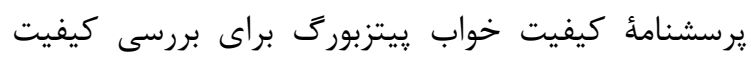

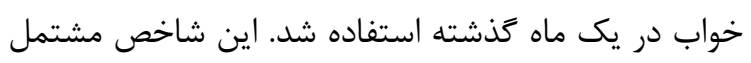

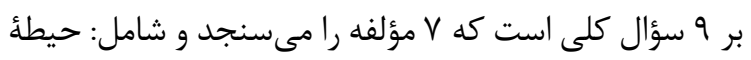

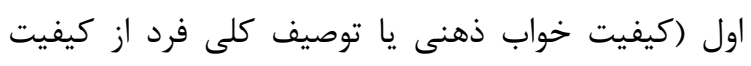

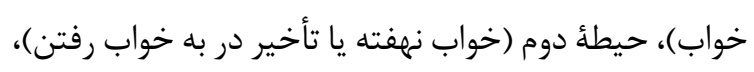

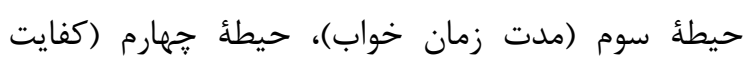

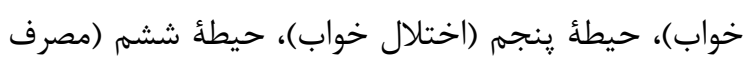

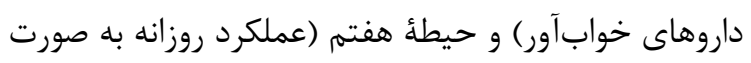

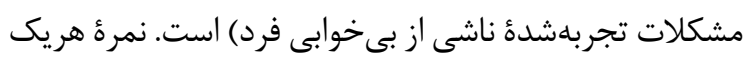

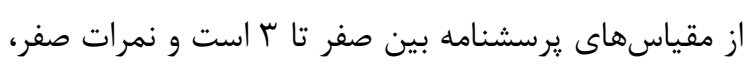

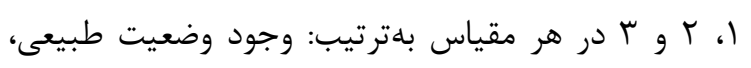

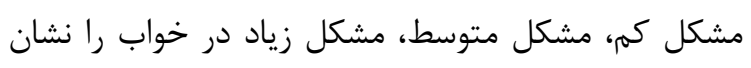

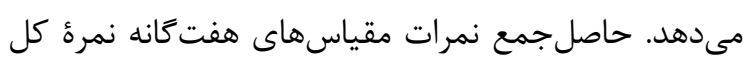

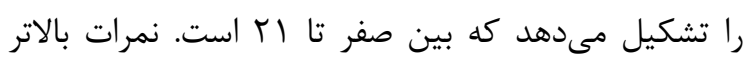
كيفيت خواب ريايينتر و نمرة بالاتر از ه ه كيفيت خواب

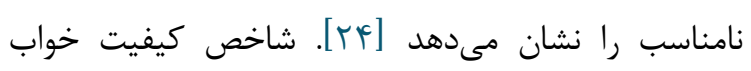

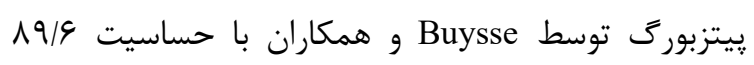

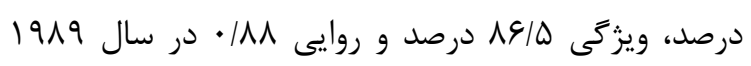

جدول ا. سياهؤ مربوط به جلسات مراقبت معنوى

\begin{tabular}{|c|c|c|}
\hline تمرينات مراقبت معنوى & عنوان جلسات & جلسات \\
\hline 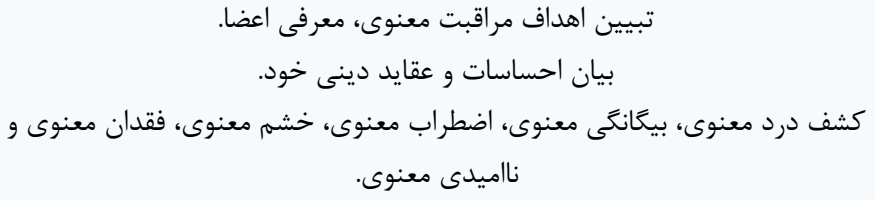 & 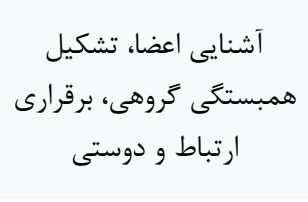 & جلسأ اول \\
\hline
\end{tabular}


F تأثير مراقبت معنوى بر كيفيت خواب بيماران مبتلا به مولتيبل اسكلروزيس F.

قرائت قرآن توسط اعضاى داوطلب گروه، همراه با ترجمهٔ آنها.

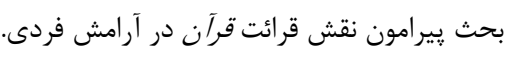

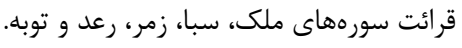

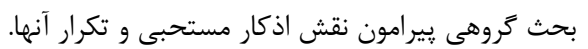

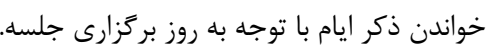

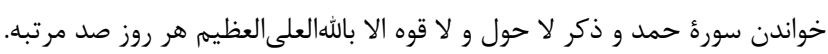

خواندن دعاى مخصوص خواب نيمساعت قبل از به خواب رفتن.

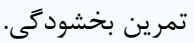

$$
\begin{aligned}
& \text { جشم:يوشى و كنترل خشم در برابر فرد خاطى. تمرئ. } \\
& \text { تمرينات مربوط به تصويرسازى. }
\end{aligned}
$$

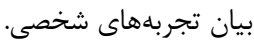

$$
\begin{aligned}
& \text { بيان عقايد. } \\
& \text { آمادهسازى اعضا براى ترى گروه. } \\
& \text { استفاده از دستاوردهاى آنها. }
\end{aligned}
$$

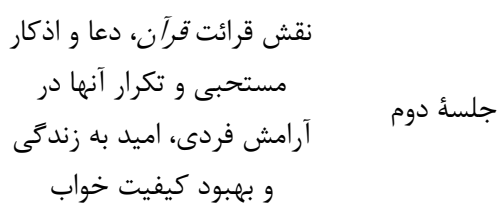

بياناتى در ارتباط با توبه،

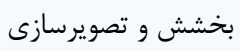
جلسؤ سوم تبيين تجربه و عقايد معنوى و استفاده از دستاوردهاى آنها توبن
با توجه به جدول ₹ آزمون تى مستقل نشان مىدهد كه دو

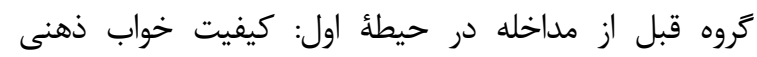

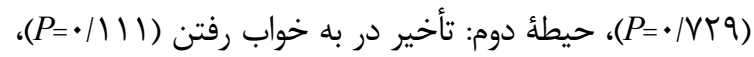

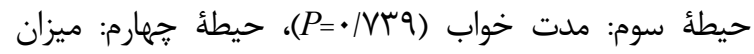

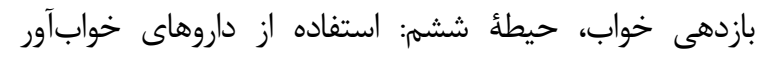

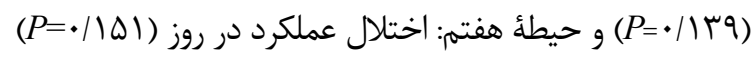
تفاوت آمارى معنادارى نداشتند، اما بعد از مداخله همهٔ حيطهها،

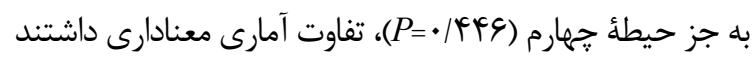

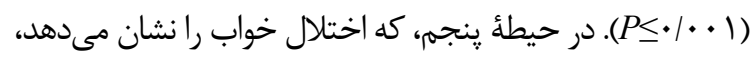

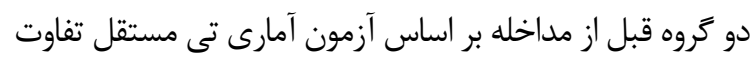

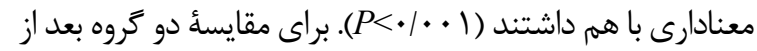

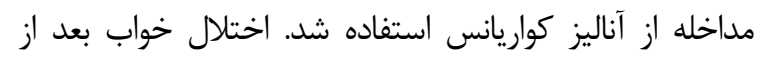

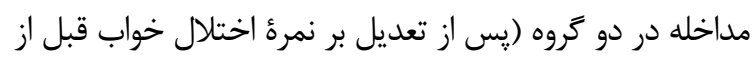

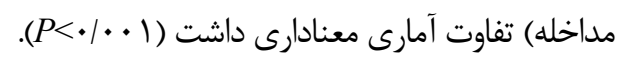

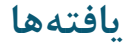
بر اساس آزمون تى مستقل دو گروه آزمون و كنترل از نظر

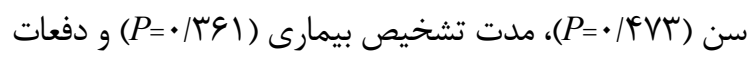

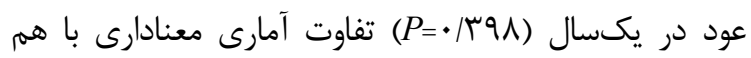
نداشتند و دو گروه از نظر اين خصوصيات دموكرافيك كمى دانى همگن بودند (جدول ץ). بر اساس آزمون دقيق فيشر دو خروه

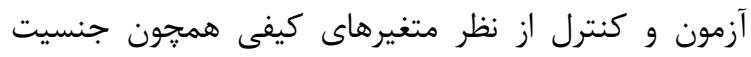
(

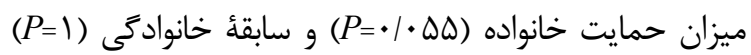
همكَن بودند و تفاوت آمارى معنادارى نداشتند. همجنين نتايج

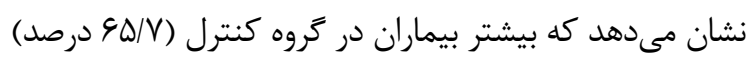

\begin{tabular}{|c|c|c|c|c|c|c|c|}
\hline$P$ value & df & $\mathbf{t}$ & ميانكين & انحراف معيار & ت تعداد & تروه & متغير \\
\hline \multirow{2}{*}{.$/ A V T$} & \multirow{2}{*}{91} & \multirow{2}{*}{$1 / \Delta \Lambda$} & $r N \cdot r$ & $9 / 19$ & ra & آزمون & \multirow{2}{*}{ سن (سال) } \\
\hline & & & $r F / Q F$ & $N \& V$ & ro & كنترل & \\
\hline \multirow{2}{*}{.$|r 9|$} & \multirow{2}{*}{91} & \multirow{2}{*}{. 199.} & $r / F \Lambda$ & $1 / \pi 9$ & ro & آزمون & \multirow{2}{*}{ مدت تشخيص } \\
\hline & & & T/Tr & .194 & ro & كنترل & \\
\hline \multirow{2}{*}{.$/ 491$} & \multirow{2}{*}{91} & \multirow{2}{*}{ - /NAT } & r & $1 / 11$ & ro & آزمون & \multirow{2}{*}{ دفعات عود در } \\
\hline & & & $r / T$. & •/Ar & ro & كنترل & \\
\hline
\end{tabular}

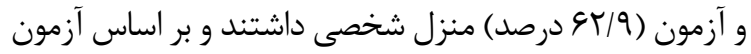

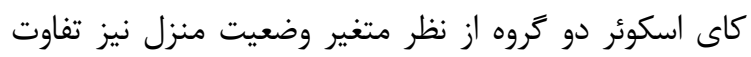

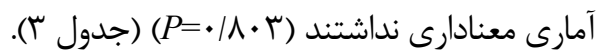


جدول r. مقايسٔ كروهها از نظر متغيرهاى كيفى

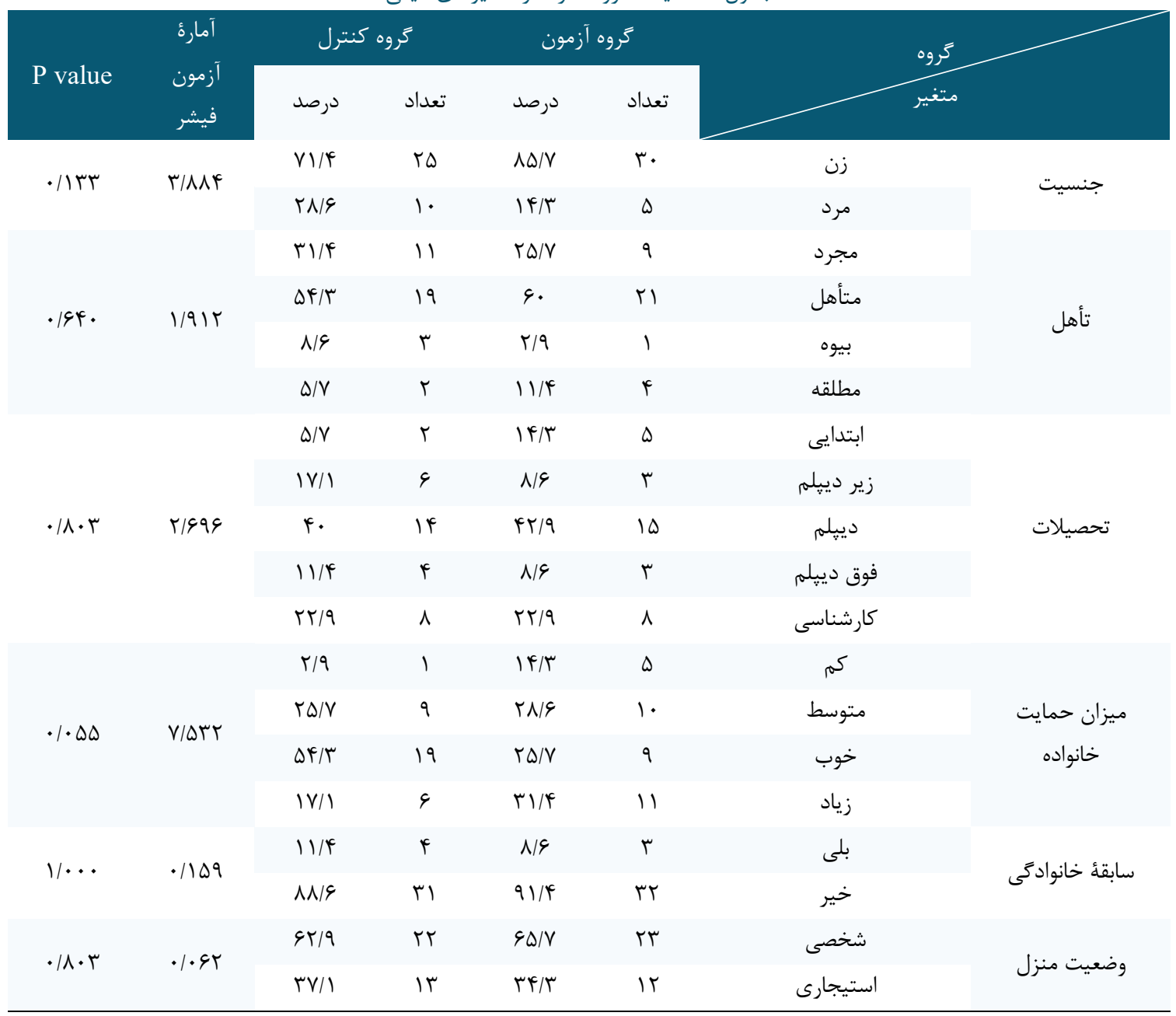

جدول f. ميانكَين و انحر اف معيار نمرؤ حيطههاى مختلف كيفيت خواب قبل و بعد از مداخله در دو كروه آزمون و كنترل

\begin{tabular}{|c|c|c|c|c|c|c|c|c|}
\hline حيطة هفتم & حيطهُ ششم & حيطة بنجم & حيطه جهارم & حيطلة سوم & حيطة دوم & حيطة اول & \multicolumn{2}{|c|}{ متغير } \\
\hline انحراف & انحراف & انحراف & انحراف & انحراف & انحراف & انحراف & & زم \\
\hline معيار土ميانگين & معيار \#ميانكَين & معيارئميانكين & معيار ¥ميانكَين & معيار土ميانگين & معيار ¥ميانكَين & معيار \#ميانكين & & \\
\hline $1 / \mu Y_{ \pm} \cdot / V q$ & $\cdot / 99 \pm \cdot / 1 \mathrm{~V}$ & $|/ \Delta| \pm \cdot|\Delta|$ & $1 / 4 r \pm 1 / r q$ & $1 / q \cdot \pm 1 / \cdot r$ & $1 / V Y \pm \cdot / 9 V$ & $1 / V Y \pm . / 99$ & $\begin{array}{l}\text { آزمون } \\
\text { كروه }\end{array}$ & \\
\hline $1 / 11 \pm \cdot / \Delta r$ & $1 / \cdot r \pm 1 / \cdot r$ & $1 / \cdot 9 \pm \cdot /{ }^{F}$ & $1 / / f \pm 1 / r$. & $1 / 99 \pm 1 / 11$ & $\tau / 1 / \pm \cdot / \Lambda$. & $1 / 99 \pm \cdot / V T$ & كنترل & مداخله از \\
\hline$\cdot|| \Delta \mid$ & . 1149 & $<\cdot|\cdot \cdot|$ & . & . Nrq & .1111 & . $/ V Y q$ & $P$ & \\
\hline $.199 \pm . / 4 \lambda$ & . & $\cdot / 9 q \pm \cdot / K F$ & $1 / r \varphi \pm 1 / r T$ & $\cdot / \Lambda \uparrow \pm \cdot / \Lambda q$ & $\cdot|V F \pm| 9 \mid$. & $\cdot \mid 99 \pm . / 4 \lambda$ & آزمون & \\
\hline $1 / 11 \pm \cdot / \Delta r$ & $1 / r \cdot \pm 1 / \cdot r$ & $1 / \cdot 9 \pm \cdot / r \Lambda$ & $1 / \cdot r \pm 1 / r V$ & $1 / 99 \pm 1 / 11$ & $\Gamma / 1 / \pm \cdot / \Lambda$. & $1 / 99 \pm \cdot / V 1$ & كنترل & مداخله \\
\hline$<\cdot|\cdot \cdot|$ & $<\cdot|\cdot \cdot|$ & $\cdot / \cdot 1$ & . kF\& & $\cdot / \cdot 1$ & $<\cdot / \cdot \cdot 1$ & $<\cdot|\cdot \cdot|$ & $P$ & \\
\hline
\end{tabular}




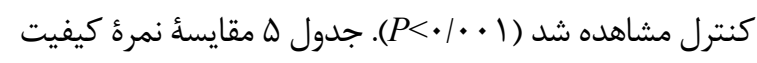

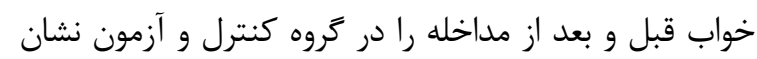

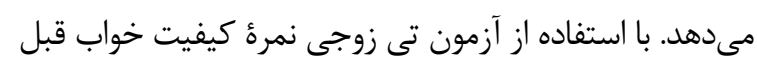

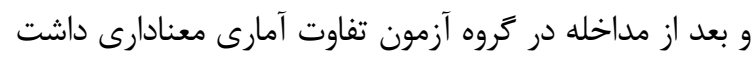

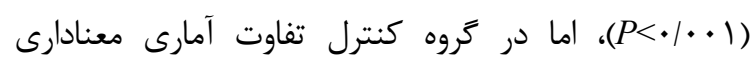

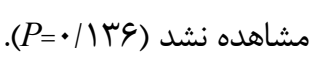

جدول ه مقايسٔ نمرة كل كيفيت خواب را در دو كروه

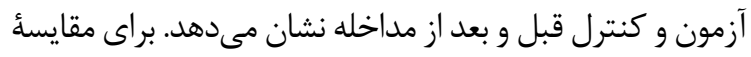
نمره كيفيت خواب دو كروه آزمون و كنترل قبل و بعد بعد از مداخ مداخله

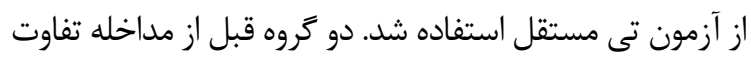

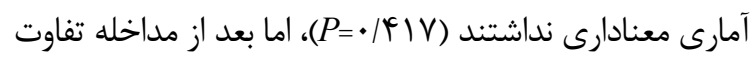

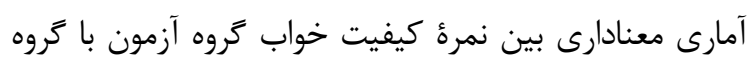

جدول ه. ميانكَين و انحراف معيار نمرةٔ كل كيفيت خواب قبل و بعد از مداخله در دو كروه آزمون و كنترل

\begin{tabular}{|c|c|c|c|c|c|}
\hline \multirow{2}{*}{$P$ value } & \multirow{2}{*}{$\mathbf{t}$} & \multirow{2}{*}{ df } & بعد از مداخله & قبل از مداخله & \\
\hline & & & انحراف معيار \ميانكَين & انحراف معيار \ميانكَين & \\
\hline$<\cdot|\cdot \cdot|$ & V/VIF & rq & $1 \cdot / V V \pm 1 / V V$ & $\mid r / V Y \pm r / \Lambda \Lambda$ & كروه آزمون \\
\hline \multirow[t]{3}{*}{ - } & $-1 / \Delta T A$ & ry & $|r / \varepsilon q \pm r /| V$ & $\Delta I / I r \pm T / r F$ & كروه كنترل \\
\hline & & & $-9 / 19 \mathrm{~V}$ & $\cdot / 4 \mid V$ & $\mathrm{t}$ \\
\hline & & & $<\cdot|\cdot \cdot|$ & $\cdot \mid 9 \vee \Lambda$ & $P$ value \\
\hline
\end{tabular}

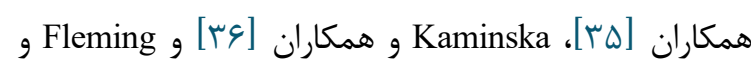

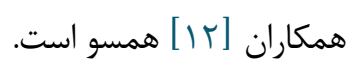

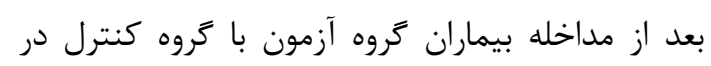

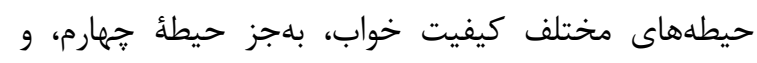
همجنين از نظر كيفيت خواب كلى با يكديكر اختلاف

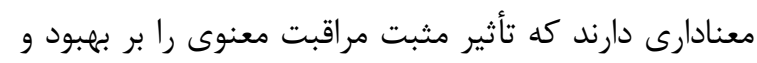

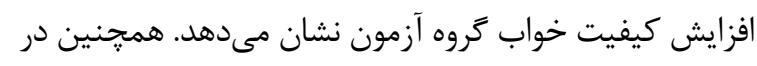

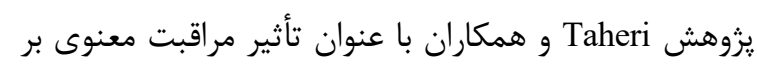
اساس مدل قلب سليم بر كيفيت خواب بيماران عروق كرونر

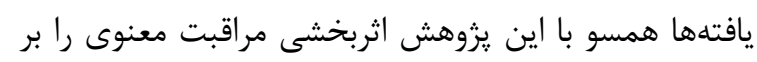

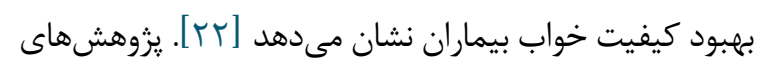

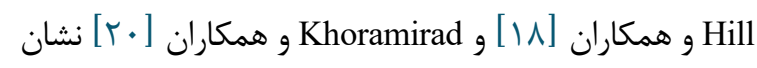
مى دهد كه دخالت مذهبى بر بهبود كيفيت خواب إن بيماران

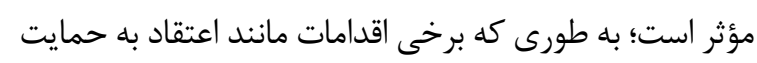

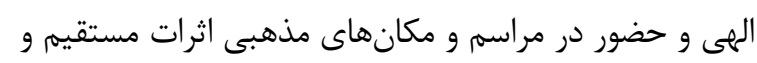

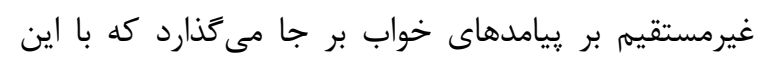
يزوهش همسو است. يزوهش Phillips و همكاران اهميت

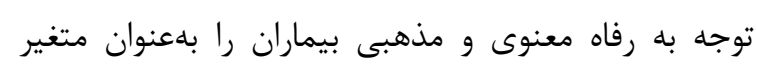

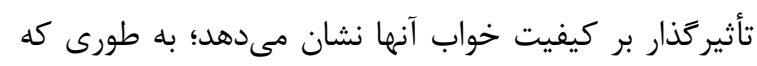

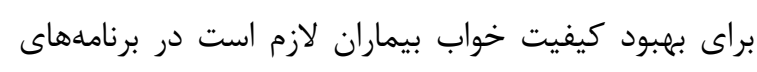

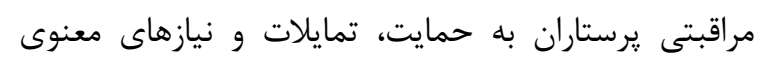

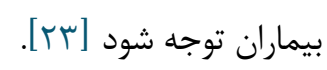

هدف اين يزوهش تأثير مراقبت معنوى بر كيفيت خواب

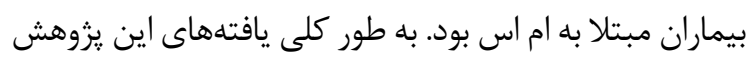

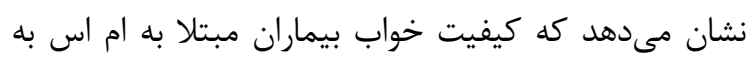
دنبال مداخلئ مراقبت معنوى به صورت مؤثر بهببود مى يابد.

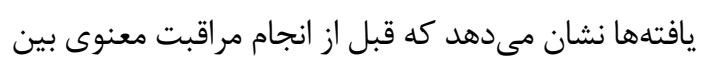
كيفيت خواب كلى بيماران كروه آزمون (با ميانكَين و انحراف

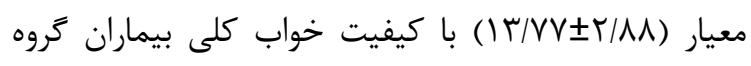

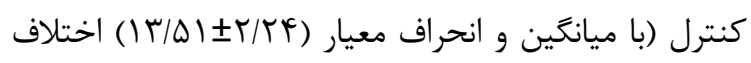

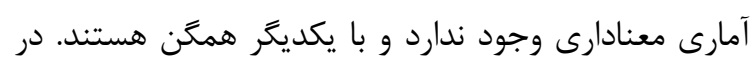

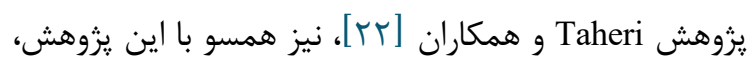

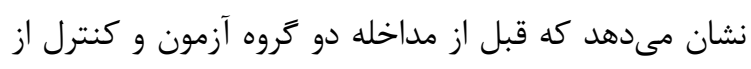
نظر كيفيت خواب كلى تفاوت آمارى معنادارى با يكديكر دان

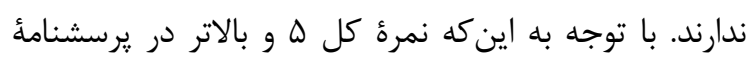

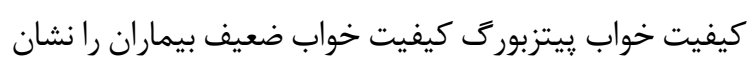

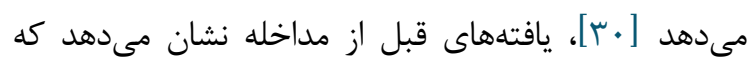

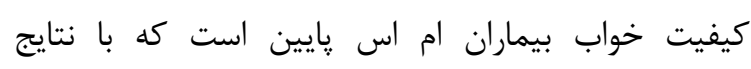

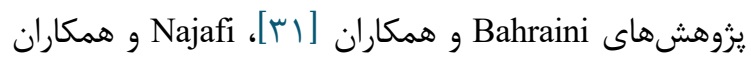

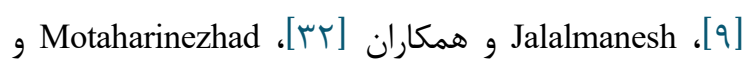

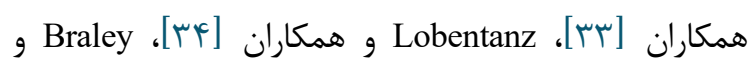


بيماران ام اس است كه به كاهش عملكرد زندكى، از هم كسيختكى خانواده و ناتوانى در انجام مسئوليتهاى فردى الهي،

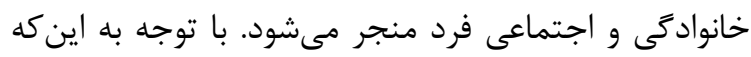

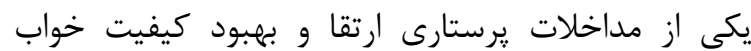

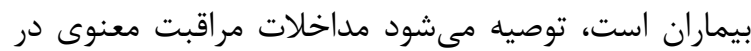
برنامههاى درمانى بيماران مبتلا ام اس به كار ترفتيه شود.$$
\text { سياسگزارى }
$$

اين مقاله بركرفته از باياننامهٔ كارشناسى ارشد يرستارى

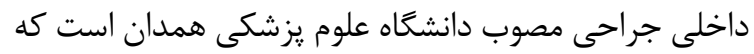

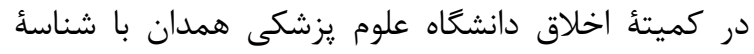

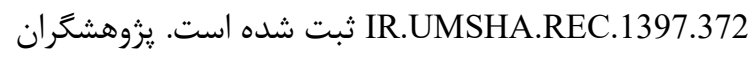
از معاونت محترم تحقيقات و فناورى دانشعاه، به دليل بروان

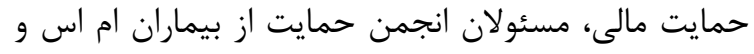

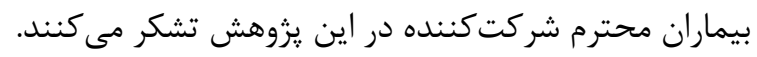

$$
\text { تعارض در منافع }
$$

بين نويسندًان هيجَّونه تعارضى در منافع وجود ندارد

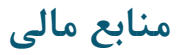

منابع مالى اين مطالعه توسط نويسندًان تامين شده است.

\section{References}

1. Moslem Khani M, Ebrahimi ME, Sahebi A. The Effectiveness of Acceptance and Commitment Therapy (ACT) on Happiness and Hope of Hamedanian Multiple Sclerosis Patients. Pajouhan Scientific Journal. 2019 Mar 15;17(2):15-23. [DOI:10.21859/psj.17.2.15]

2. Hosseini SM, Asgari A, Rassafiani M, Yazdani F, Mazdeh M. Leisure Time Activities of Iranian Patients with Multiple Sclerosis: a Qualitative Study. Health promotion perspectives. 2016;6(1):47. [DOI:10.15171/hpp.2016.08] [PMID] [PMCID]

3. Seighalani M, Birshak B, Mohammadizadeh A. The Effectiveness of Music Therapy on Depression and Anxiety of Patients with Multiple Sclerosis. Psychological studies. 2015;11(4):7-22.

4. Nasiri M, Hosseini H, Sakhaei Y, Tabrizi N, Yazdani Cherati J, Abedini M. Prevalence of Psychiatric Disorders in Patients with Multiple Sclerosis in Mazandaran, Iran. Journal of Mazandaran University of Medical Sciences. 2016 Sep 15;26(140):60-70.
يروهش Yang و همكاران با عنوان تأثير مراقبت معنوى بر كيفيت خواب بيماران همودياليزى نشان مى دهد بين فعاليتهاى مذهبى و معنوى با كيفيت خواب كلى بيماران تفاوت آمارى معنادارى وجود ندارد كه با اين يزوهش تناقض دارد و ممكن است به دليل تفاوت بين فرهنگ مذهبى، زمينه و نوع بيمارى در

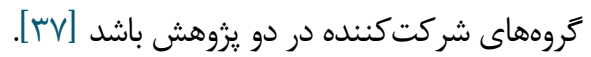

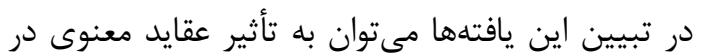

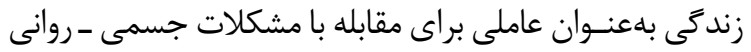

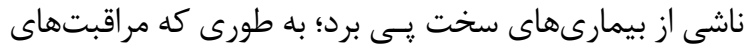

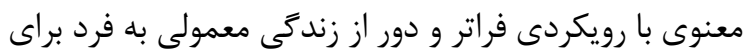

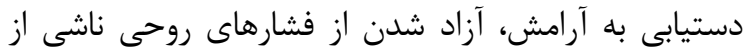

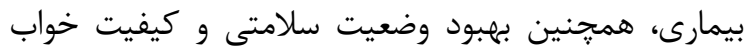

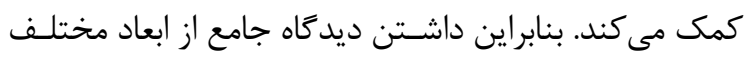

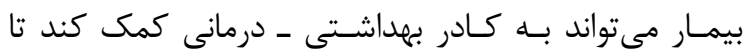
خدمات مناسبترى بـهـ بيمـاران مبـتلا بـهـ بيمارىهاى مزمن

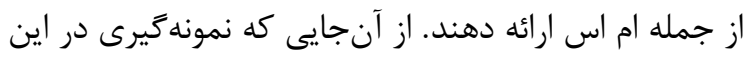

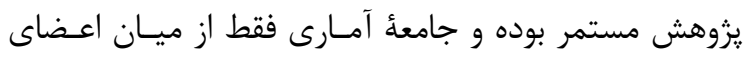

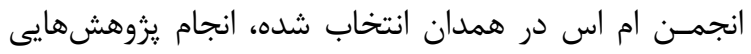
مشابه در شهر همدان و ديخر شهرها يِيشنهاد مىشود.

$$
\text { نتيجه گيبرى }
$$

مراقبت معنوى در بيماران ام اس كيفيت خواب بيماران

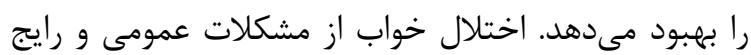

5. Rabin BS. Can Stress Participate in the Pathogenesis of Autoimmune Disease?. Journal of adolescent health. 2002 Apr 1;30(4):71-5. [DOI:10.1016/S1054139X(01)00384-6]

6. Moreira NC, Damasceno RS, Medeiros CA, De Bruin PF, Teixeira CA, Horta WG, De Bruin VM. Restless Leg Syndrome, Sleep Quality and Fatigue in Multiple Sclerosis Patients. Brazilian Journal of Medical and Biological Research. 2008 Oct;41(10):932-7. [DOI:10.1590/S0100-879X2008001000017] [PMID]

7. Bamer AM, Johnson KL, Amtmann D, Kraft GH. Prevalence of Sleep Problems in Individuals with Multiple Sclerosis. Multiple Sclerosis Journal. 2008 Sep;14(8):1127-30. [DOI:10.1177/1352458508092807] [PMID] [PMCID]

8. Hughes AJ, Turner AP, Alschuler KN, Atkins DC, Beier M, Amtmann D, Ehde DM. Association between Sleep Problems and Perceived Cognitive Dysfunction Over 12 Months in Individuals with Multiple Sclerosis. Behavioral sleep medicine. 2018 Jan 2;16(1):79-91.

[DOI:10.1080/15402002.2016.1173553] [PMID] 
Fץ تأثير مراقبت معنوى بر كيفيت خواب بيماران مبتلا به مولتييل اسكلروزيس

9. Najafi MR, Etemadifar M, Haghighi S, Maghzi AH, Akbari M. The Study of Circadian Rhythms Disorder and Its Relationship with Chronic Fatigue in Multiple Sclerosis in Compare with Control Group. Journal of Isfahan Medical School. 2011 Jan 3;28(113).

10. Sack RL, Auckley D, Auger RR, Carskadon MA, Wright Jr KP, Vitiello MV, Zhdanova IV. Circadian Rhythm Sleep Disorders: Part I, Basic Principles, Shift Work and Jet Lag Disorders. Sleep. 2007 Nov 1;30(11):1460-83. [DOI:10.1093/sleep/30.11.1460] [PMID] [PMCID]

11. Vancampfort D, De Hert M, Knapen J, Maurissen K, Raepsaet J, Deckx S, Remans S, Probst M. Effects of Progressive Muscle Relaxation on State Anxiety And Subjective well-being in People with Schizophrenia: A Randomized Controlled Trial. Clinical Rehabilitation. 2011 Jun;25(6):567-75. [DOI:10.1177/0269215510395633] [PMID]

12. Fleming WE, Pollak CP. Sleep Disorders in Multiple Sclerosis. In Seminars in neurology 2005 Mar (Vol. 25, No. 01, pp. 64-68). Copyright $(2005$ by Thieme Medical Publishers, Inc., 333 Seventh Avenue, New York, NY 10001, USA. [DOI:10.1055/s-2005867075] [PMID]

13. Akbari Z, Mirzaei M, Azizi R. The Quantity and Quality of Sleep and Their Relationship to Obesity. Journal of Mazandaran University of Medical Sciences. 2016 Jun 15;26(137):128-36.

14. Mazaheri M, Falahi KM, Sadat MS, Rahgozar M. Nursing Attitude to Spirituality and Spiritual Care. Payesh. 2009;8(1):31-7.

15. Rahimi N, Nouhi E, Nakhaee N. Spiritual well-being and Attitude toward Spirituality and Spiritual Care in Nursing and Midwifery Students. Iran Journal of nursing. 2013 Dec;26(85):55-65.

16. Selman L, Harding R, Gysels M, Speck P, Higginson IJ. The Measurement of Spirituality in Palliative Care and the Content of tools Validated Cross-culturally: a Systematic Review. Journal of pain and symptom management. 2011 Apr 1;41(4):728-53. [DOI:10.1016/j.jpainsymman.2010.06.023] [PMID]

17. Oshvandi K, Amini S, Moghimbeigi A, Sadeghian E. The Effect of Spiritual Care on Death Anxiety in Hemodialysis Patients with End-stage of Renal Disease: A Randomized Clinical Trial. Journal of hayat. 2018 Jan 15;23(4):332-44.

18. Hill TD, Deangelis R, Ellison CG. Religious Involvement as a Social Determinant of Sleep: An Initial Review and Conceptual Model. Sleep health. 2018 Aug 1;4(4):325-30. [DOI:10.1016/j.sleh.2018.04.001] [PMID]

19. Kaheni S, Heidar-Far J, Nasiri E. Relationship between Spiritual Intelligence and Medicaldemographic Characteristics in Community-dwelling Elderly. Journal of Mazandaran University of Medical Sciences. 2013 Jun 15;23(101):87-94.

20. Khoramirad A, Mousavi M, Dadkhahtehrani T, Pourmarzi D. Relationship between Sleep Quality and Spiritual Well-being/religious Activities in Muslim Women with Breast Cancer. Journal of religion and

\section{health. $2015 \quad$ Dec 1;54(6):2276-85 [DOI:10.1007/s10943-014-9978-0] [PMID]}

21. Atef-vahid MK, Nasr-Esfahani M, Esfeedvajani MS, Naji-Isfahani H, Shojaei MR, Masoumeh YM, Goushegir SA. Quality of Life, Religious Attitude and Cancer Coping in a Sample of Iranian Patients with Cancer. Journal of research in medical sciences: the official journal of Isfahan University of Medical Sciences. 2011 Jul;16(7):928.

22. Saeidi Taheri Z, Asad Zandi M, Ebadi A. The effect of spiritual care based on Ghalbe Salim Model on the sleep quality of the patients with coronary artery disease. Iran J Crit Care Nurs. 2014; 7(2):92-101

23. Phillips KD, Mock KS, Bopp CM, Dudgeon WA, Hand GA. Spiritual Well-being, Sleep Disturbance, and Mental and Physical Health Status in HIV-infected Individuals. Issues in Mental Health Nursing. 2006 Jan 1;27(2):125-39. [DOI:10.1080/01612840500436917] [PMID]

24. Mirghafourvand M, Mohammad-AlizadehCharandabi S, Mansouri A, Najafi M, Khodabande F. The Effect of Vitamin D and Calcium Plus Vitamin D on Sleep Quality in Pregnant Women with Leg Cramps: A Controlled Randomized Clinical Trial. Journal of Isfahan Medical School. 2015;32(320):2444-53.

25. Buysse DJ, Reynolds CF, Monk TH, Berman SR, Kupfer DJ. The Pittsburgh Sleep Quality Index: a New Instrument for Psychiatric Practice and Research. Psychiatry res. 1989 May 1;28(2):193-213. [DOI:10.1016/0165-1781(89)90047-4]

26. Hosseinabadi R, Norouzi K, Pouresmaeil Z, Karimlou M, Sadat MS. Acupoint Massage in Improving Sleep Quality of Older Adults. Archives of Rehabilitation. 2008;9(2):8-14.

27. Soleimany M, Ziba FN, Kermani A, Hosseini FA. Comparison of Sleep Quality in two Groups of Nurses with and without Rotation Work Shift Hours. Iran Journal of Nursing. 2007 Apr;20(49):29-38.

28. Cheawchanwattana A, Chunlertrith D, Saisunantararom W, Johns NP. Does the Spiritual Well-Being of Chronic Hemodialysis Patients Differ from that of Pre-dialysis Chronic Kidney Disease Patients?. Religions. 2015 Mar;6(1):14-23. [DOI:10.3390/rel6010014]

29. Mahdavi B, Fallahi Khoshknab M, Mohammadi F, Hosseini MA. The effect of Group Spiritual Care on Quality of Life in Family Caregivers of Elders with Alzheimer's Disease. Journal of health promotion management. 2015 Jun 10;4(3):34-42.

30. Liao JL, van den Broek-Best O, Smyth B, Hong D, Vo K, Zuo L, Gray NA, Chan CT, de Zoysa J, Perkovic V, Jiang L. Effect of Extended Hours Dialysis on Sleep Quality in a Randomized Trial. Nephrology. 2019 Apr;24(4):430-7. [DOI:10.1111/nep.13236] [PMID]

31. Bahraini S, Bekhradi R, Mannani R, Naji SA. The Effect of Massage Therapy on the Quality of Sleep in Women with Multiple Sclerosis being Admitted by Isfahan MS Association. The Journal of Urmia Nursing and Midwifery Faculty. 2011;8(4):197-203. 
32. Jalalmanesh SH, Zargarani F. Effects of Progressive Muscle Relaxation Technique on Fatigue and Sleep Quality in Patients with Multiple Sclerosis. Scientific Journal of Hamadan Nursing \& Midwifery Faculty (Nasim-Danesh). 2015;23(3):5-14.

33. Motaharinezhad F, Parvaneh S, Bakhtiary AH, Alizadeh N, Ghahari S. The Effect of Mood and Cognition on Relationship between Sleep Disturbances and Fatigue in People with Multiple Sclerosis. Koomesh. 2016 Jan 19:613-9.

34. Lobentanz IS, Asenbaum S, Vass K, Sauter C, Klösch G, Kollegger H, Kristoferitsch W, Zeitlhofer J. Factors Influencing Quality of Life in Multiple Sclerosis Patients: Disability, Depressive Mood, Fatigue and Sleep Quality. Acta Neurologica Scandinavica. 2004 Jul;110(1):6-13. [DOI:10.1111/j.16000404.2004.00257.x] [PMID]

35. Braley TJ, Boudreau EA. Sleep disorders in multiple sclerosis. Current neurology and neuroscience reports. 2016 May 1;16(5):50. [DOI:10.1007/s11910-0160649-2] [PMID]

36. Kaminska M, Kimoff RJ, Schwartzman K, Trojan DA. Sleep Disorders and Fatigue in Multiple Sclerosis: Evidence for Association and Interaction. Journal of the neurological sciences. 2011 Mar 15;302(1-2):7-13. [DOI:10.1016/j.jns.2010.12.008] [PMID]

37. Yang JY, Huang JW, Kao TW, Peng YS, Lu CS, Chen DL, Yang CS, Yang CC, Tsai DM, Liao CS, Chang HW. Impact of Spiritual and Religious Activity on Quality of Sleep in Hemodialysis Patients. Blood purification. 2008;26(3):221-5. [DOI:10.1159/000118845] [PMID] 